\title{
High sensitivity phonon spectroscopy of Bose-Einstein condensates using matter-wave interference
}

\author{
N. Katz ${ }^{1}$, R. Ozeri ${ }^{1 *}$, J. Steinhauer ${ }^{1 \dagger}$, N. Davidson ${ }^{1}$, C. Tozzo ${ }^{2,3}$ and F. Dalfovo ${ }^{3,4}$ \\ ${ }^{1}$ Department of Physics of Complex Systems, Weizmann Institute of Science, Rehovot 76100, Israel \\ 2 Dipartimento di Fisica, Università di Trento, I-38050 Povo, Italy \\ 3 Istituto Nazionale per la Fisica della Materia, BEC-INFM Trento, I-38050 Povo, Italy \\ ${ }^{4}$ Dipartimento di Matematica e Fisica, Università Cattolica del Sacro Cuore, Via Musei 41, 25121 Brescia
}

\begin{abstract}
We study low momentum excitations of a Bose-Einstein condensate using a novel matter-wave interference technique. In time-of-flight expansion images we observe strong matter-wave fringe patterns. The fringe contrast is a sensitive spectroscopic probe of in-trap phonons and is explained by use of a Bogoliubov excitation projection method applied to the rescaled order parameter of the expanding condensate. Gross-Pitaevskii simulations agree with the experimental data and confirm the validity of the theoretical interpretation. We show that the high sensitivity of this detection scheme gives access to the quantized quasiparticle regime.
\end{abstract}

The peculiar nature of a Bose-Einstein condensate (BEC) is clearly observed in its low momentum excitations, revealing the many-body coherent nature of the system. Recent experiments have provided observations of the Bogoliubov excitation spectrum, superfluidity, and suppression of low momentum excitations due to quantum correlations in the ground state 1, 2, 3].

In these experiments the condensates were typically excited by Bragg pulses [4] and imaged after a free expansion. At high momenta the excitations were clearly separated from the expanding condensate, and could be counted and quantified. Even in the phonon regime (wavelength larger than but comparable to the healing length) a well defined excitation cloud could still be distinguished from the condensate (see Fig. 1a) and was found to be amenable to direct atom counting methods.

However, at sufficiently low momentum (wavelength much larger than the healing length), we observe a new regime, in which the excitations and the condensate no longer separate, regardless of the duration of the expansion. The excitations are manifested in a clear density modulation of the cloud in the absorption images (see Fig. 1c), reminiscent of in-situ observations of low energy, zero momentum, excitations of the condensate [6, 7], but they differ significantly from these since they involve both nontrivial time-of flight (TOF) dynamics and a small, but finite, excitation momentum.

In this Letter we analyze the dynamics of phonons in such freely expanding condensates using both the GrossPitaevskii equation (GPE) and a dynamically rescaled Bogoliubov theory [8]. We find that, in our elongated condensate, axial low-momentum phonons are adiabatically converted by the (mainly radial) expansion into free

\footnotetext{
${ }^{*}$ Current address: Time and Frequency Division NIST 325 Broadway Boulder, Colorado 80305, USA

${ }^{\dagger}$ Current address: Department of Physics, Technion - Israel Institute of Technology, Technion City, Haifa 32000, Israel
}

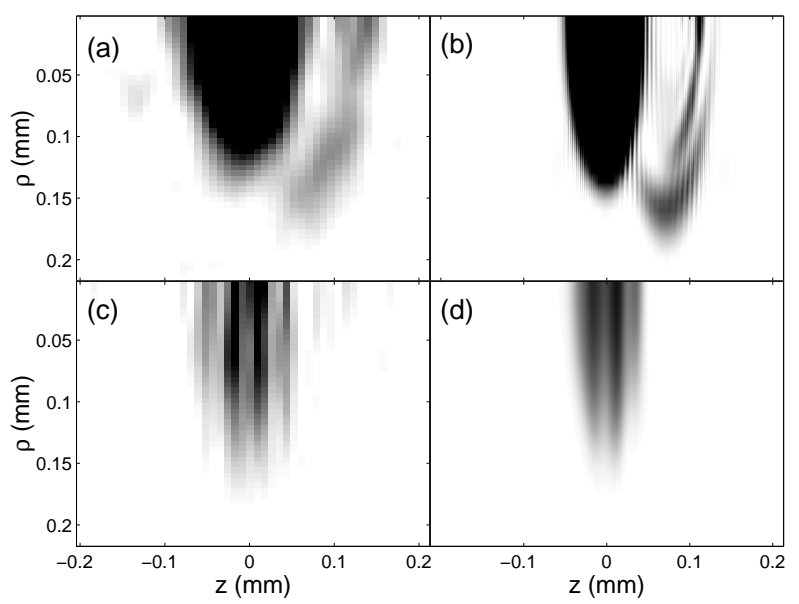

FIG. 1: Density $n(\rho, z)$ of the expanding condensate, initially excited with phonons of momentum $q$. In (a) and (b): $q a_{\rho}=2.03$. The density in (a) is obtained by computerized tomography of TOF column density absorption images, $n_{\text {col }}(y, z)$, while (b) corresponds to a full GP simulation. In both cases, note the clearly separated excitation cloud on the right of the condensate. The same quantities are plotted in (c) and (d) for $q a_{\rho}=0.31$. Note the strong density modulation, with no significant outcoupled fraction.

atoms with the same axial momentum. The overlap of these moving free particles with the expanding ground state results in the axial periodic density modulations observed in the TOF images.

We use the fringe visibility of these density modulations after TOF, as an extremely sensitive spectroscopic probe of the excitation strength. Whereas the sensitivity of atom or momentum counting methods [2] scales quadratically with the quasiparticle excitation amplitude, we show that the fringe visibility scales linearly with these amplitudes. This enhanced sensitivity was noted and used to estimate the degree of adiabaticity in the loading of a BEC into an optical lattice [9], but not as a spectroscopic probe of the excitations themselves. 
Our nearly pure $(>95 \%){ }^{87} \mathrm{Rb}$ condensate in the $\left|F, m_{f}\right\rangle=|2,2\rangle$ ground state, is formed in an elongated cylindrically-symmetric harmonic trap with axial frequency $\omega_{z}=2 \pi \times 25 \mathrm{~Hz}$ and radial frequency $\omega_{\rho}=2 \pi \times 220 \mathrm{~Hz}$. The radial harmonic oscillator length is $a_{\rho}=\left(\hbar / m \omega_{\rho}\right)^{1 / 2}=0.73 \mu \mathrm{m}$. We use condensates with $N=10^{5}$ atoms, having chemical potential $\mu=\eta \hbar \omega_{\rho}$, with $\eta=9.1$, and an average healing length $\xi \sim 0.3 a_{\rho}$. Using Bragg excitation beams detuned $6.5 \mathrm{GHz}$ above the $5 S_{1 / 2}, F=2 \longrightarrow 5 P_{3 / 2}, F^{\prime}=3$ transition, Bogoliubov quasiparticles are excited in the condensate along the $\hat{z}$ axis, at a controlled wavenumber $q$ by varying the angle between the two beams. At a given $q$ the frequency difference between the beams $\omega$ is controlled via acoustooptic modulators. After the Bragg pulse (of duration $t_{B}=6 \mathrm{msec}$ ) the magnetic trap is rapidly turned off and after $38 \mathrm{msec}$ of TOF an on-resonant absorption image is taken, with the absorption beam perpendicular to the $\hat{z}$-axis.

The dynamics of phonons within an expanding condensate has recently been studied [8] and shown that, within a short time interval $\left(t \sim \omega_{\rho}^{-1}\right)$, phonons are converted into single particle excitations travelling at an axial velocity of the order of $\hbar q / m$. This velocity should be compared to that of the slowly expanding axial boundary, which travels at the asymptotic velocity $\pi \omega_{z}^{2} Z_{T F} / 2 \omega_{\rho}$, where $Z_{T F}=a_{\rho}\left(\omega_{\rho} / \omega_{z}\right)(2 \eta)^{1 / 2}$ is the Thomas-Fermi axial radius of the condensate. Thus at long expansion times the excitations can separate out from the condensate only if $q a_{\rho}>q_{c} a_{\rho}=\pi\left(\omega_{z} / \omega_{\rho}\right)(\eta / 2)^{1 / 2}$, while for $q<q_{c}$ they remain within the condensate at all times. For our experimental parameters one has $q_{c} a_{\rho}=0.76$. Experimentally, this estimate is remarkably exact. Indeed, we begin to observe a distinct excitation cloud at $q a_{\rho} \simeq 0.72$. However, this transition is not sharp and a small fraction still overcomes the condensate boundary even at lower $q$. This escaped fraction was used to find the resonance in our previous measurement of the excitation spectrum [2].

In the phonon regime, but with $q>q_{c}$, using computerized tomography [5] (see Fig. 1a), the freely expanding excitation cloud was measured to be carrying almost all the momentum and excess energy of the system due to excitation. By solving the GPE for the TOF dynamics, we now confirm this experimental observation quantitatively for this regime. For instance, the lateral cloud in Fig. $1 \mathrm{~b}$ carries about $99 \%$ of the momentum and more than $95 \%$ of the energy of the initial phonons. In Fig. $1 \mathrm{~b}$ one also notices that the released cloud is radially wider than the condensate, in agreement with the experimental observation [5] and has an evident "shell structure". Both features are associated with the spatial structure of the Bogoliubov amplitudes of the in-trap initial excitations [8] and the presence of excitations with radial nodal lines. At this momentum $\left(q a_{\rho}=2.03\right)$ counting the population of the excitation cloud also gives reason-

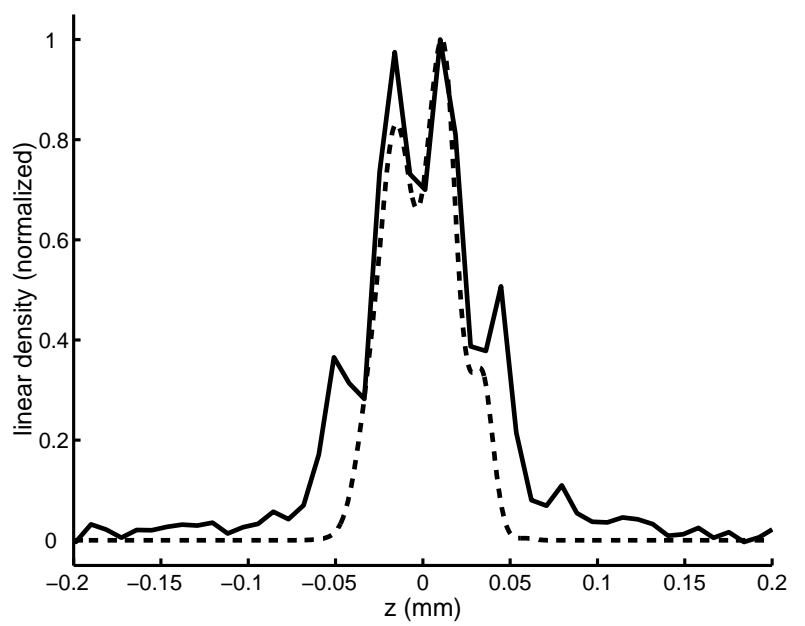

FIG. 2: Linear density $2 \pi \int_{0}^{\infty} \rho n(\rho, z) d \rho$ measured from TOF image with very low momentum excitation $q a_{\rho}=0.31$ (solid line). Note the slight asymmetry to right, which is in agreement with the GPE simulation (dashed line).

able agreement with the population expected by simple structure factor considerations [2].

Turning our attention to the very low momentum excitations with $q a_{\rho}=0.31$, we study the TOF density modulations of Figs. 1c and 1d in detail. Fig. 2 shows the linear density of the expanded condensate, defined as $2 \pi \int_{0}^{\infty} \rho n(\rho, z) d \rho$. We note a slight asymmetry to the right, which is related to the initial direction of propagation of the phonon 10 .

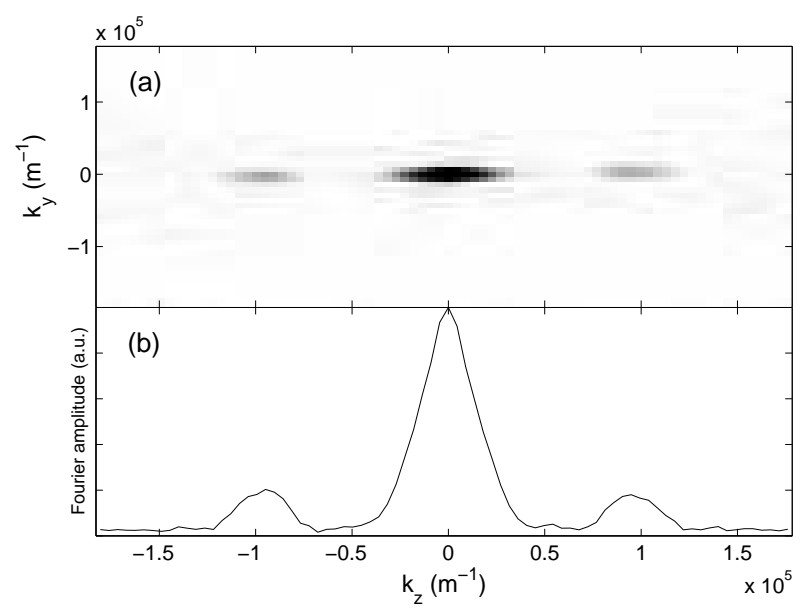

FIG. 3: Fourier transform of the column density $n_{\mathrm{col}}(y, z)$ for the same image as Fig. 1c. (a) Fourier transform. (b) Integration over central strip of (a). Note the clear separation between the central mode and the sidelobes, despite the low momentum of the excitation.

To quantify the fringes of Fig. 1c, we take the Fourier transform of the measured density profile, and observe clear sidelobes (Fig. 3). We define the fringe visibility as 
the area of the sidelobes divided by the area of the central lobe. This is a powerful "spatial lock-in" method to reduce noise in the measurement. It also agrees strictly with the standard definition of contrast. We note the ease of the measurement, due to the clear separation between the Fourier peaks, despite the very low momenta involved. This should be contrasted with the atom counting methods, where it becomes increasingly difficult to discern the excitation cloud at finite expansion times in real space. At such low momentum we do not expect any significant radial excitations, since the coupling to all higher radial modes is predicted to be negligible [11, 12]. This is confirmed by the lack of any features in the transverse direction in Figs. 1c and 3a.

Fig. 4 shows the fringe visibility as a function of the Bragg frequency detuning (circles). We find the resonances to be at $\pm 137 \pm 10 \mathrm{~Hz}$, which compares favorably with the expected value $138 \pm 5 \mathrm{~Hz}$ for the average Bogoliubov frequency in local density approximation (LDA) [16]. The width of the resonances is due to the finite duration of the Bragg pulse.

A direct GPE simulation of the experiment (Fig. 4, solid line) gives good agreement with the observed data [17. However, little intuition is gained by this. Therefore, we also compare this spectrum to the analytic result obtained with a rescaled quasiparticle projection method [8] (dashed line).

For this, we consider a uniform gas in a box of volume $L^{3}$, with a density $n_{0}=N / L^{3}$. The ground state order parameter is $\Psi_{0}=\sqrt{n_{0}}$, with chemical potential $\mu=g n_{0}$. The excited states are the well known Bogoliubov quasiparticles, having frequency $\omega_{k}=$ $\left\{k^{2} /(2 m)\left[\hbar^{2} k^{2} /(2 m)+2 \mu\right]\right\}^{1 / 2}$ and amplitudes $u_{k}, v_{k}=$ $\pm\left\{\left[\hbar^{2} k^{2} /(2 m)+2 \mu\right] /\left(2 \hbar \omega_{k}\right) \pm 1 / 2\right\}^{1 / 2}$.

We insert the linear expansion

$$
\begin{aligned}
\Psi(z, t)= & e^{-i \mu t / \hbar}\left\{n_{0}^{1 / 2}+L^{-3 / 2} \sum_{k} c_{k}(t) u_{k} e^{i\left(k z-\omega_{k} t\right)}\right. \\
& \left.+c_{k}^{*}(t) v_{k}^{*} e^{-i\left(k z-\omega_{k} t\right)}\right\}
\end{aligned}
$$

into the GPE. The Bragg pulse is included through the potential $V=V_{B} \cos (q z-\omega t)$, acting for $-t_{B}<t<0$. At $t=-t_{B}$ the condensate is assumed to be in the ground state, so that $c_{k}\left(-t_{B}\right)=0$ for all $k$ 's. The equations for the coefficients $c_{k}(t)$ are then solved analytically $[12,13]$. After the Bragg pulse one gets

$$
c_{ \pm q}(0)=\mp \frac{V_{B} \sqrt{N}\left(u_{q}+v_{q}\right)}{2 \hbar\left(\omega \mp \omega_{q}\right)}\left(e^{ \pm i\left(\omega \mp \omega_{q}\right) t_{B}}-1\right),
$$

and $c_{k}(0)=0$ for $|k| \neq q$.

Next we assume that the uniform gas undergoes a fictitious radial expansion which mimics the actual expansion, by taking a uniform time-dependent density that decreases in time as the average density of elongated condensate. For this we choose the density $n_{0}$ to be equal to the radial average of the density of a cylindrical condensate, $n_{0}=(2 / 5) n(0)$, having the same central density $n(0)$ of our trapped condensate, and we treat the expansion by means of the scaling ansatz 14, 15.

In this case, the density decreases as $n_{0} / b^{2}(t)$, where $b^{2}(t)=1+\omega_{\rho}^{2} t^{2}$ is the radial scaling parameter. In the presence of quasiparticles with wavevector $\pm q$, one can write a rescaled order parameter $\tilde{\Psi}$ in the form

$\tilde{\Psi}(z, t)=\tilde{\Psi}_{0}+L^{-3 / 2} \sum_{k= \pm q} c_{k}(0) \tilde{u}_{k}(t) e^{i k z}+c_{k}^{*}(0) \tilde{v}_{k}^{*}(t) e^{-i k z}$

At $t=0$, when $\tilde{u}_{k}(0)=u_{k}, \tilde{v}_{k}(0)=v_{k}$ and $\tilde{\Psi}_{0}=\sqrt{n_{0}}$, this expression coincides with (11). At $t>0, \tilde{\Psi}_{0}$ remains stationary while the quantities $\tilde{u}$ and $\tilde{v}$ are timedependent amplitudes that obey rescaled Bogoliubov-like equations [8].

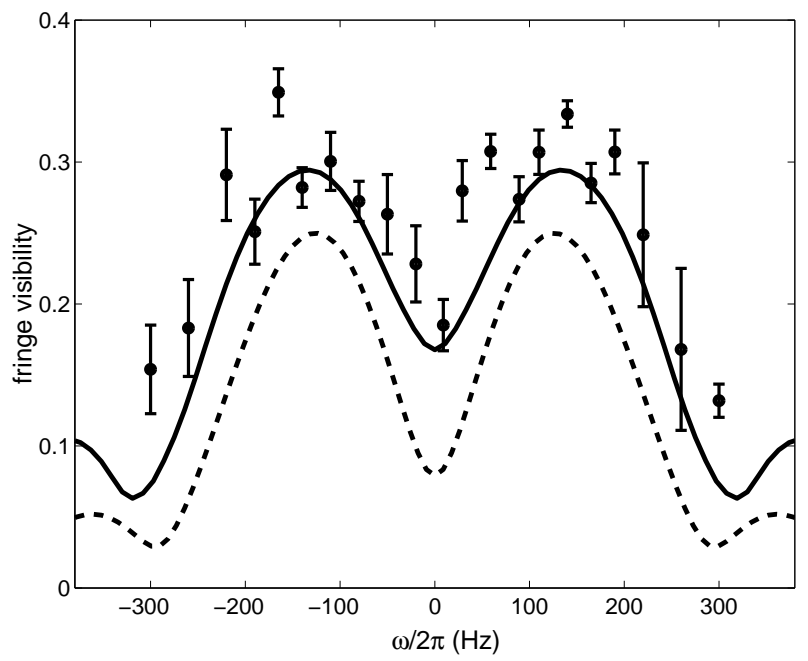

FIG. 4: Fringe visibility as a function of the Bragg excitation frequency (points). The error bars represent the uncertainty due to four measurements. We observe a clear doublepeaked spectrum, which is finite-time broadened. The peaks are found at $\omega= \pm 137 \pm 10$, close to the expected Bogoliubov frequency $(138 \pm 5 \mathrm{~Hz})$. The solid line is a full GPE simulation of the experiment. The dashed line is the result of Eq. (5).

Further simplification is obtained by assuming adiabatic quasiparticle-to-particle conversion. This corresponds to assuming that, after an expansion time $t \gg$ $\omega_{\rho}^{-1}$, the rescaled amplitude $\tilde{u}$ becomes unity, while $\tilde{v}$ vanishes [18]. In terms of $\tilde{\Psi}$, this implies

$$
\tilde{\Psi}(z, t) \rightarrow \tilde{\Psi}_{0}+L^{-3 / 2}\left[c_{q}(0) e^{i q z}+c_{-q}^{*}(0) e^{-i q z}\right] .
$$

Since the expansion is assumed to be radial, the linear density obtained by radial integration of the rescaled density $|\tilde{\Psi}|^{2}$ coincides with that obtained from $|\Psi|^{2}$. The same is true for their Fourier components with $k=0$ 
and $k= \pm q$. The sum of the $\pm q$ coefficients, divided by the $k=0$ coefficient is exactly the visibility $\gamma$ as defined for the experimental analysis. One finds $\gamma=(2 / \sqrt{N})\left|c_{q}(0)+c_{-q}^{*}(0)\right|$ or, recalling Eq. (2),

$$
\begin{aligned}
\gamma & =\frac{V_{B}}{\hbar}\left[1+\frac{4 \mu m}{\hbar^{2} q^{2}}\right]^{-1 / 4} \\
& \times\left|\frac{e^{i\left(\omega-\omega_{q}\right) t_{B}}-1}{\omega_{q}-\omega}+\frac{e^{-i\left(\omega+\omega_{q}\right) t_{B}}-1}{\omega+\omega_{q}}\right| .
\end{aligned}
$$

The dashed line in Fig. 4 corresponds to this expression, calculated for the same $V_{B}$ used in the GPE simulations $\left(V_{B}=0.1 \hbar \omega_{\rho}\right)$ and with no fitting parameters. The agreement with both experiment and GPE simulation indicates that the essence of the release process of a BEC with such very low momentum excitations is indeed captured by simply assuming that, due to the reduction of the average background density in which the excitations live, quasiparticles are adiabatically converted into free particles that interfere with the ground state.

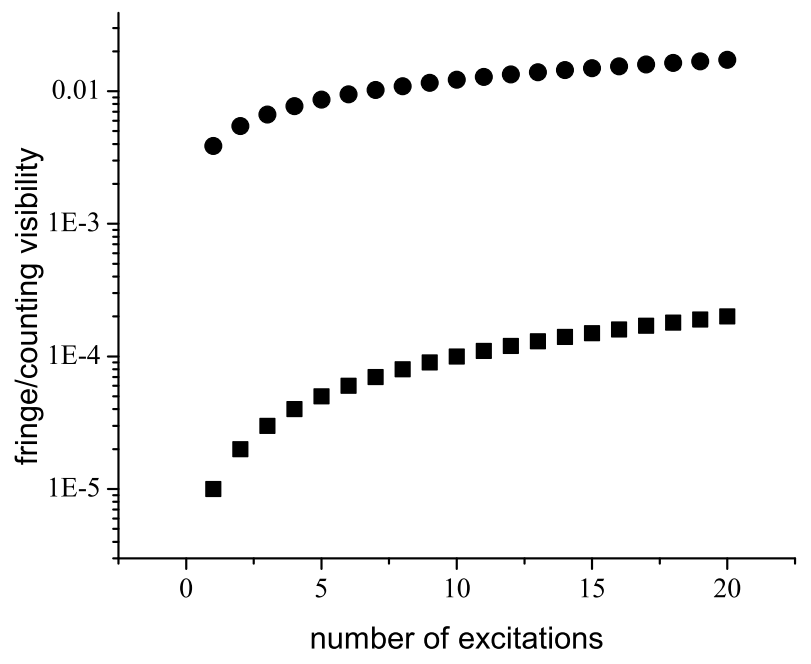

FIG. 5: Fringe visibility after TOF for $q a_{\rho}=0.31$ (circles) compared with the standard atom counting technique for $q a_{\rho}=2.03$ (boxes), as a function of the number of excitations. The visibility of the atom counting method grows linearly in excitation number with a very weak slope of $1 / N$, whereas the fringe visibility is not negligible $(\sim 0.4 \%)$ even for a singly quantized excitation, and grows as the square root of the number of excitations.

We stress that, in this linear response analysis for $q<q_{c}$, the predicted fringe visibility is proportional to $c_{q}$, which is in turn proportional to $V_{B}\left(u_{q}+v_{q}\right)$. By contrast, at higher momentum $\left(q>q_{c}\right)$, the number of observed atoms in the excitation cloud is linear in the number of excitations, given by $\left|c_{q}\right|^{2}$, and quadratic in the quasiparticle amplitudes. This is consistent with the results of our GPE simulations, where the fringe visibility at low $q$ and the counting visibility at high $q$ are found to be proportional to $V_{B}$ and $V_{B}^{2}$, respectively.
In our experiments and simulations, we typically excite $\sim 10^{2}$ quasiparticles. However, by extrapolating the expected fringe visibility to lower Bragg intensities, one finds that it remains sizeable even in the range of single quantized excitations. We compare (see Fig. 5) the fringe visibility at $q a_{\rho}=0.31$ (circles) with the counting visibility at $q a_{\rho}=2.03$ (boxes) for our experimental parameters 19]. The fringe visibility at low $q$ is predicted to be over two orders of magnitude more sensitive. This implies a sensitive heterodyne technique to observe few, and possibly single quasi-particle excitations.

In conclusion, we discuss and implement a novel fringe visibility technique to observe the in-situ matter-wave interference of low momentum excitations with the condensate. We measure the excitation spectrum with high resolution and analyze the possibility of singly quantized excitation detection with this technique.

This work was supported in part by the Israel Ministry of Science, the Israel Science foundation and the DIP foundation. We thank J. Steinhauer for his experimental contribution.

[1] D. M. Stamper-Kurn et al., Phys. Rev. Lett. 83, 2876 (1999).

[2] J. Steinhauer et al., Phys. Rev. Lett. 88, 120407 (2002).

[3] W. Ketterle and S. Inouye, Comptes Rendus de l'Academie des Sciences 2, 339 (2001).

[4] J. Stenger et al., Phys. Rev. Lett. 82, 4569 (1999); Phys. Rev. Lett 84, 2283 (2000).

[5] R. Ozeri et al., Phys. Rev. Lett. 88, 220401 (2002)

[6] M.-O. Mewes et al., Phys. Rev. Lett. 77, 988 (1996).

[7] D. S. Jin et al., Phys. Rev. Lett. 77 420, (1996).

[8] C. Tozzo and F. Dalfovo, e-print cond-mat/0401359

[9] J. Hecker Denschlag et al., J. Phys. B,35, 3095 (2002).

[10] We note a discrepancy in the axial size of the expanded condensate as compared to the GPE results, probably due to experimental imperfections in the release process. The effect on the radial expansion and on the experimental observables discussed here is negligible.

[11] J. Steinhauer et al., Phys. Rev. Lett. 90, 060404 (2003).

[12] C. Tozzo and F. Dalfovo, New J. Phys. 5, 54 (2003).

[13] P.B. Blakie, R.J. Ballagh, and C.W. Gardiner, Phys. Rev. A 65, 033602 (2002).

[14] Y. Kagan, E.L. Surkov and G.V. Shlyapnikov, Phys. Rev. A 54, R1753 (1996); Phys. Rev. A 55, R18 (1997).

[15] Y. Castin and R. Dum, Phys. Rev. Lett. 77, 5315 (1996).

[16] A. Brunello et al., Phys. Rev. A. 64, 063614 (2001).

[17] In the experimental data (and GP simulation) of Fig. 4 we note an offset due to background signal and a small overlap of the sidelobes with the central lobe in Fourier space.

[18] In the case of the expanding homogeneous gas model adiabaticity has been shown to fail for $q a_{r}<q_{a} a_{r} \sim \eta^{-1 / 2}$ 8], corresponding to $q a_{r}<0.3$. However, numerical analysis of the expanding inhomogeneous system indicates that adiabaticity fails only at an even lower $q$.

[19] The counting visibility at high $q$ is defined as the ratio 
between the number of counted atoms in the excitation cloud, which is equal to the number of quasiparticles, and the total number of atoms $N$. 